\title{
Aspectos Biométricos del Canal Mandibular
}

\author{
Biometric Aspects of the Mandibular Canal \\ *Iván Claudio Suazo Galdames; **Carlos Andrés Morales Herrera; \\ "Mario Gonzalo Cantín López \& *** Daniela Alejandra Zavando Matamala
}

SUAZO, G. I. C.; MORALES, H. C.A.; CANTÍN, L. M. G. \& ZAVANDO, M. D. A. Aspectos biométricos del canal mandibular. Int. J.Morphol., 25(4):811-816, 2007.

RESUMEN: El canal mandibular recorre este hueso longitudinalmente, relacionándose en su segmento medio con las piezas dentarias molares y premolares. El conocimiento de la relación del canal mandibular con las piezas dentarias y con las corticales mandibulares es de gran importancia para la práctica de la implantología. En el presente estudio se analizan las relaciones de la porción media del canal mandibular con las corticales mandibulares pertenecientes a individuos de sexo femenino, de entre 50 y 65 años, con caracterización antropométrica coincidente con el registro. Se realizaron mediciones en tres niveles del segmento medio (A-B-C). Los resultados muestran que el canal mandibular presenta una forma ovoídea y que sus diámetros disminuyen a medida que desciende hasta el agujero mentoniano. Se observó además, un trayecto oblicuo y descendente, aproximándose a la cortical vestibular de la mandíbula. Se discute la importancia del conocimiento de las características biométricas del canal mandibular, en individuos de sexo femenino, del grupo etario seleccionado, debido a que estos son pacientes quienes tienen mayor necesidad de rehabilitación con implantes óseointegrados.

\section{PALABRAS CLAVE: Anatomía; Biometría; Canal mandibular.}

\section{INTRODUCCIÓN}

La anatomía mandíbular ha sido descrita por una gran cantidad de autores (Rouvière \& Delmas, 1999; Figún \& Garino, 2001; Latarjet \& Ruiz-Liard, 2004). La mandíbula se encuentra formada por un hueso basal que constituye su mayor parte, presta origen a la musculatura y soporta al hueso alveolar, encargado éste de contener las piezas dentarias con las cuales articula (Misch, 1994, 2006; Lindhe, 2005).

La mandíbula contiene en su interior, un canal que la recorre parcialmente en sentido longitudinal, denominado canal mandibular, que nace en la cara medial de la rama de la mandíbula en el foramen mandibular, protegido por una língula, y luego desciende por el tejido esponjoso del hueso en sentido anteroinferior, siguiendo una curva de concavidad anterosuperior (Rouviere \& Delmas; Figún \& Garino; Latarjet \& Ruiz-Liard).

El canal mandibular puede ser dividido en tres segmentos: uno posterior, oblicuo anteroinferiormente, que comprende desde la língula mandibular hasta el segundo molar; uno medio, que tiende a ser horizontal y se acerca a la tabla vestibular y al borde basilar, se extiende desde el segundo molar hasta el segundo premolar; y otro anterior que comienza a nivel del segundo premolar donde se bifurca originando el canal mentoniano y el canal incisivo, el cual luego se pierde en el espesor del tejido esponjoso del cuerpo mandibular.

En los cortes coronales, el canal mandibular tiene un aspecto de círculo u óvalo de eje mayor vertical, donde destaca una cortical ósea que demarca su periferia.

Reiser et al. (2004) describen que en su porción media, el canal mandibular se encuentra a $6,59 \mathrm{~mm}$ del borde basilar y presenta un diámetro de 3,3 mm. En el área del foramen mentoniano, el canal se localiza a $8,91 \mathrm{~mm}$ del borde basilar y presenta un diámetro de $3,2 \mathrm{~mm}$.

El canal mandibular puede presentar diversas variaciones anatómicas que tienen importancia clínica; Olivier en 1927 (Op cit. Girard, 1979) describió que en el 60\% de los casos, el canal mandibular es un verdadero túnel con

\footnotetext{
* Unidad de Anatomía Normal, Facultad de Ciencias de la Salud, Universidad de Talca, Chile.

* Cirujano Dentista, práctica privada.

*** Departamento de Estomatología, Facultad de Ciencias de la Salud, Universidad de Talca, Chile.
} 
paredes corticalizadas gruesas, que se adelgazan más y más a medida que se acercan al final del recorrido; mientras que el $40 \%$ restante, es mucho menos individualizado y que en el hueso esponjoso, es un pasaje más que un túnel.

En sentido transversal, el canal mandibular es más próximo a la tabla lingual y medial en relación a las raíces del tercer y segundo molares, subyacente al ápice del primer molar y vestibularizándose respecto al segundo premolar (Figún \& Garino).

Carter \& Keen (1971) describen tres tipos de posición del canal mandibular, Tipo I: el canal mandibular situado muy cerca de las raíces dentarias; Tipo II: el canal mandibular está situado inferior a las raíces de los molares mandibulares, las conexiones del canal mandibular con las raíces dentarias son, por lo tanto, más largas y oblicuas; Tipo III: el canal mandibular se encuentra más posterior e inferior que en los tipos anteriores. Estos autores además, indican que el Tipo I, es mucho más común que los Tipos II y III, en una relación de $75 \%$ el Tipo I, un $12.5 \%$ para el Tipo II y $12.5 \%$ el Tipo III.

La localización correcta del canal mandibular es una parte fundamental en la planificación de la rehabilitación de un paciente desdentado mandibular, mediante implantes oseointegrados, para lo cual se utiliza comúnmente la radiografía panorámica, siendo este recurso de una validez limitada, debido a que es una imagen bidimensional y con distorsión. La tomografía computarizada es el examen adecuado para la visualización el canal mandibular, ésta presenta $94 \%$ de exactitud, mientras que la radiografía periapical un $53 \%$ y las imágenes panorámicas 17\% de exactitud (Reiser).

El estudio busca aportar conocimientos de los parámetros biométricos para la localización del canal mandibular en cada uno de sus segmentos, en un grupo de mandíbulas de mujeres de entre 50 y 65 años.

\section{MATERIAL Y MÉTODO}

Se utilizaron 26 mandíbulas pertenecientes al Departamento de Anatomía Normal de la Universidad de Talca, pertenecientes a individuos de sexo femenino de entre $50 \mathrm{y}$ 65 años, obtenidas de distintos cementerios de las regiones VI y VII, con caracterización antropométrica coincidente con el registro (Kondo \& Townsed, 2004; Kemkes-Grottenthaler et al., 2002).

Al momento de determinar los puntos para realizar las mediciones, esto no se logró en la totalidad de los seg- mentos a analizar, debido a diversos factores como, la fractura de alguna cortical en el momento del corte, principalmente la cortical basal; falta de algún segmento de la cortical que conforma el canal mandibular, segmentos en los cuales las corticales del canal mandibular se confundían con el tejido óseo esponjoso. Se trazó una línea guía perpendicular a la base de la mandíbula, que tocaba tangencialmente al borde anterior del foramen mentoniano de la hemimandíbula izquierda. A 1 centímetro de la línea guía, paralela a ésta, se establece la primera línea de corte, denominando a este segmento como, segmento A. Luego a 1 centímetro de la primera línea de corte y paralela a ésta, se traza la segunda línea de corte, llamando a este segmento, segmento B. Posteriormente a 1 centímetro de la segunda línea de corte y paralela a ésta, se traza la tercera y última línea de corte, llamando a este segmento, segmento C.

El corte se realizó con una Sierra de banda eléctrica de corte vertical (Labre Armand ${ }^{\circledR}$ Motor de 2HP y 1400 RPM, huincha de corte de $1 \mathrm{~mm}$ de ancho) por la líneas previamente trazadas, quedando 3 trozos por mandíbula (Fig. 1).

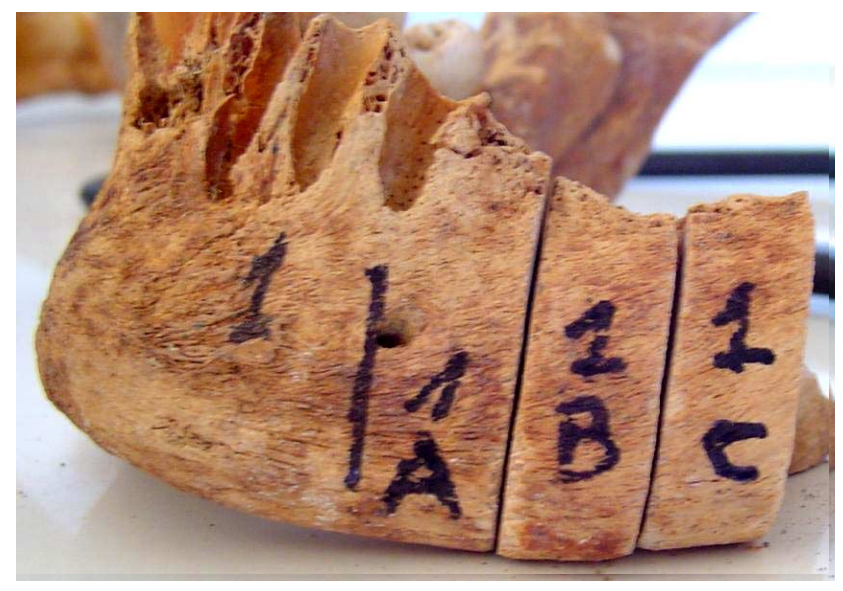

Fig. 1. Fotografía de mandíbula $\mathrm{N}^{\mathrm{o}} 1$ con línea guía y segmentos A, B y C, después del corte.

Posteriormente, se realizaron mediciones con un pie de metro en las porciones distales de los segmentos A, B y C, obteniéndose: el diámetro vertical máximo del canal mandibular, el diámetro horizontal máximo del canal mandibular, la distancia del canal mandibular a la cortical vestibular, la distancia del canal mandibular a la cortical lingual, la distancia del canal mandibular al borde basilar (Fig 2).

La información recopilada fue ordenada en el programa Microsoft Excel 2003, para el sistema operativo Windows XP y se obtuvieron los estadísticos descriptivos de la muestra. 

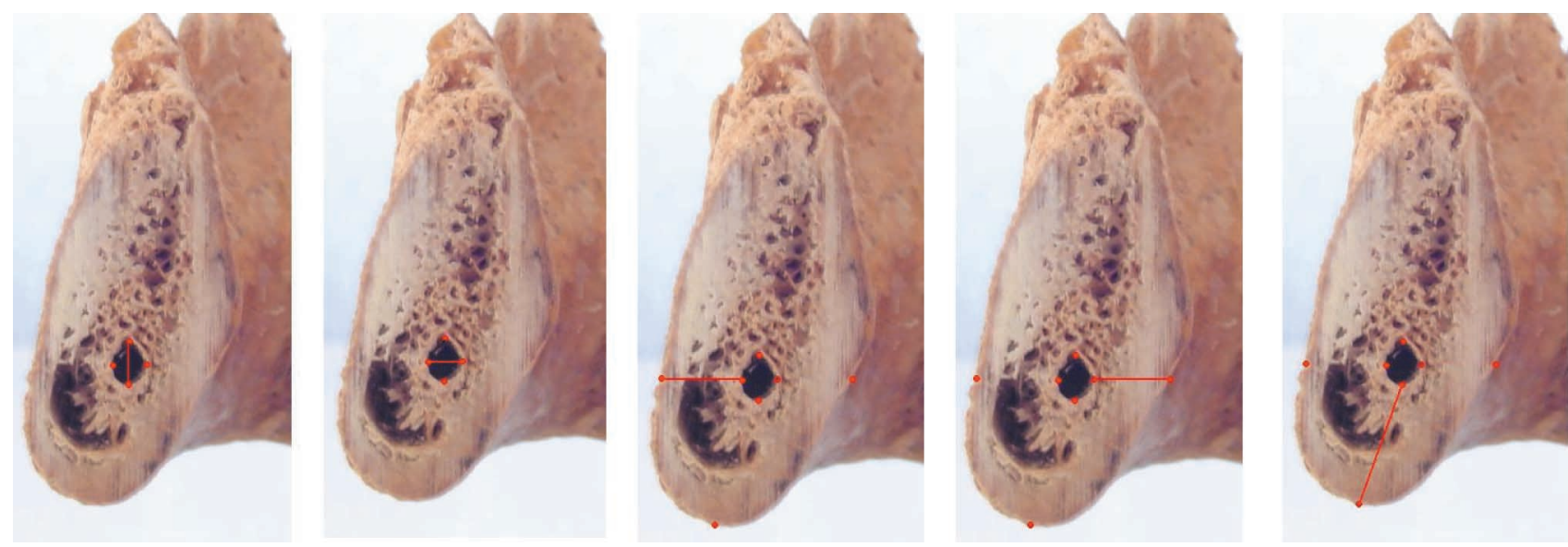

Fig. 2. A. Diámetro vertical máximo del canal mandibular; B. Diámetro horizontal máximo del canal mandibular; C. Distancia del canal mandibular a la cortical vestibular; D. Distancia del canal mandibular a la cortical lingual; E. Distancia del canal mandibular al borde basilar.

\section{RESULTADOS}

Fueron analizados 78 segmentos de 26 mandíbulas en total y a continuación se describen los resultado encontrados para cada segmento. Como se verá, en algunos segmentos no fue posible realizar todas las mediciones propuestas, ya que en algunos casos el hueso se encontraba deteriorado en el segmento analizado o bien el canal mandibular carecía de cortical en esa porción, en concordancia por lo descrito por Oliver (op cit. Girard, 1979).

Segmento A: Para determinar el diámetro vertical máximo del canal mandibular se realizaron 19 mediciones, siendo el valor promedio de $2,24 \mathrm{~mm}$ (DS 0,49), el valor máximo de 3,4 mm y el mínimo de $1,5 \mathrm{~mm}$.

Para determinar el diámetro horizontal máximo del canal mandibular se realizaron 19 mediciones, el valor promedio fue de 2, $613 \mathrm{~mm}$ (DS 0,615), con un valor máximo de 4,3 $\mathrm{mm}$ y un valor mínimo de $1,55 \mathrm{~mm}$.

Para la determinación de la distancia entre el canal mandibular y la cortical vestibular se realizaron 19 mediciones, el valor promedio fue de 3,95 mm (DS 1,05), la distancia menor fue de $2.1 \mathrm{~mm}$ y la distancia mayor fue de $5.65 \mathrm{~mm}$.

Para determinar la distancia entre el canal mandibular y el borde basilar se realizaron 19 mediciones, el valor promedio fue de 7,65 mm (DS 1,25), la distancia menor fue de $5.25 \mathrm{~mm}$ y la distancia mayor fue de $10 \mathrm{~mm}$.

Para determinar la distancia entre el canal mandibular y la cortical lingual se realizaron 19 mediciones, el valor promedio fue de $2,9 \mathrm{~mm}$ (DS 0,73), la distancia menor fue de $1,7 \mathrm{~mm}$ y la distancia mayor fue de $4.1 \mathrm{~mm}$.

Segmento B. Para la determinación del diámetro vertical máximo del canal mandibular se realizaron 19 mediciones, el valor promedio fue de 2,27 mm (DS 0,53), el valor máximo fue de $4 \mathrm{~mm}$ y el mínimo de $1,95 \mathrm{~mm}$.

Para determinar el diámetro horizontal máximo del canal mandibular se realizaron 22 mediciones, el valor promedio fue de $2,28 \mathrm{~mm}$ (DS 0,38), con un valor máximo de $3,0 \mathrm{~mm}$ y un valor mínimo de $1,4 \mathrm{~mm}$.

Para calcular la distancia entre el canal mandibular y la cortical vestibular se realizaron 20 mediciones, el valor promedio fue de 5,2 $\mathrm{mm}$ (DS 1,37), la distancia menor fue de $2,2 \mathrm{~mm}$ y la distancia mayor fue de $8,4 \mathrm{~mm}$.

Para determinar la distancia entre el canal mandibular y el borde basilar se realizaron 20 mediciones, el valor promedio fue de 7,01 $\mathrm{mm}$ (DS 1,56), la distancia menor fue de $3,8 \mathrm{~mm}$ y la distancia mayor fue de $9,8 \mathrm{~mm}$.

Para calcular la distancia entre el canal mandibular y la cortical lingual se realizaron 23 mediciones, el valor promedio fue de $2,56 \mathrm{~mm}$ (DS 0,86 ), la distancia menor fue de $1,0 \mathrm{~mm}$ y la distancia mayor fue de $4,0 \mathrm{~mm}$.

Segmento C. Para la deterimación del diámetro vertical máximo del canal mandibular se realizaron 22 mediciones, el valor promedio fue de $2,85 \mathrm{~mm}$ (DS 0,43), el valor máximo fue de 3,7 $\mathrm{mm}$ y el mínimo de 2,15 $\mathrm{mm}$. 
Tabla I. Valores promedio de cada medición del canal mandibular, por segmento, de 26 mandíbulas de individuos adultos (entre 50 y 65 años) de sexo femenino, pertenecientes al Departamento de Anatomía Normal de la Universidad de Talca.

\begin{tabular}{lccc}
\hline \multicolumn{1}{c}{ Mediciones } & Segmento A & Segmento B & Segmento C \\
Diámetro vertical máximo del canal mandibular & 2.61 & 2.77 & 2.85 \\
Diámetro horizontal máximo & 2.24 & 2.28 & 5.45 \\
Distancia entre el canal mandibular y la cortical vestibular & 3.95 & 5.9 & 7.02 \\
Distancia entre el canal mandbular y el borde basilar & 7.65 & 2.01 & 2.74 \\
Distancia entre el canal mandibular y la cortical lingual & 2.9 & 2.56 & 2.74 \\
\hline
\end{tabular}

Para determinar el diámetro horizontal máximo del canal mandibular se realizaron 22 mediciones, el valor promedio fue de $2,45 \mathrm{~mm}$ (DS 0,32 ), con un valor máximo de $3,2 \mathrm{~mm}$ y un valor mínimo de $2,0 \mathrm{~mm}$.

Para calcular la distancia entre el canal mandibular y la cortical vestibular se realizaron 21 mediciones, el valor promedio fue de 5,9 mm (DS 1,67), la distancia menor fue de $1,3 \mathrm{~mm}$ y la distancia mayor fue de $8,7 \mathrm{~mm}$.

Para determinar la distancia entre el canal mandibular y el borde basilar se realizaron 21 mediciones, el valor promedio fue de 7,02 mm (DS 1,75), la distancia menor fue de $3,1 \mathrm{~mm}$ y la distancia mayor fue de $10,25 \mathrm{~mm}$.

Para determinar la distancia entre el canal mandibular y la cortical lingual se realizaron 22 mediciones, el valor promedio fue de 2,74 mm (DS 1,16), la distancia menor fue de $1.3 \mathrm{~mm}$ y la distancia mayor fue de $5,7 \mathrm{~mm}$.

\section{DISCUSIÓN}

El canal mandibular ha sido estudiado por numerosos autores (Carter \& Keen; Farman \& Nortjé, 2002; Reiser et al.; Zachowa et al., 2006; Levine et al., 2007) y han sido descritas relaciones variables del canal mandibular, a lo largo de su trayecto con las corticales mandibulares.

Los valores promedio de los diámetros vertical y horizontal máximo del canal mandibular fueron, respectivamente, para el segmento A de 2,61 mm y 2,24 mm; segmento B de $2,77 \mathrm{~mm}$ y $2,28 \mathrm{~mm}$; y segmento $\mathrm{C}$ de $2,85 \mathrm{~mm}$ y 2,45 $\mathrm{mm}$, lo que evidencia un aumento del diámetro del canal mandibular en los planos vertical y horizontal a medida que nos aproximamos a la porción posterior del canal mandibular. Esto coincide con lo manifestado por Figún \& Garino y Reiser et al., quienes señalan que el calibre del canal mandibular declina progresivamente desde su inicio hasta su bifurcación.
La relación del canal mandibular con la cortical vestibular mandibular, en promedio, fue de $3,9 \mathrm{~mm}$ en el segmento A, 5,2 mm en el segmento B y 5,9 mm en el segmento $\mathrm{C}$, lo que evidencia una vestibularización en el trayecto del canal mandibular en su porción anterior, relación también descrita en la literatura, exponiendo el canal mandibular que en su origen se aproxima a la cortical lingual $y$, a nivel del primer molar, cruza oblicuamente, en el plano horizontal, el hueso desde medial a lateral acercándose a la cortical vestibular (Soames, 1995; Figún \& Garino).

El canal mandibular, en relación con la cortical mandibular lingual, en el segmento A tuvo un promedio de 2,9 mm, el segmento B de 2,56 mm, y el segmento $C$ de $2,74 \mathrm{~mm}$. Estos valores describen una relación más cercana del canal mandibular con la cortical mandibular lingual, lo que concuerda con lo expresado por Gowgiel (1992). Con respecto a la relación del canal mandibular con la cortical basal mandibular, los valores encontrados fueron en promedio, para el segmento A de 7,65 mm, el segmento B de 7,01 $\mathrm{mm}$ y el segmento $\mathrm{C}$ de 7,02 $\mathrm{mm}$. Esto indicaría un aumento de la distancia del canal mandibular en su relación con la cortical basal mandibular, a medida que se acerca al foramen mentoniano; lo que concuerda con el trayecto descrito por la literatura, expresando que el canal mandibular en la primera parte de su segmento medio es horizontal y se eleva a medida que se acerca al foramen mentoniano (Figún \& Garino). El análisis de los resultados de este estudio también coincide con lo expresado por Carter \& Keen, quienes describieron que el canal mandibular se encuentra en el 75\% de los casos más cercano a los ápices de las piezas dentarias que a la cortical basal de la mandíbula. Otro aspecto importante de destacar es que en la literatura se describe que el punto más cercano a la cortical basal mandibular, o punto declive del trayecto del canal mandibular, se ubica a nivel del primer molar (Figún \& Garino; Saralaya \& Narayana, 2005) concordando con los resultados de este estudio.

Por último, en este estudio se analizaron los aspectos biométricos del canal mandibular en un grupo de 
mandíbulas de mujeres de 50 a 65 años de edad, que corresponde justamente al grupo etáreo que epidemiológicamente más requiere la rehabilitación mediante implan- tes óseointegrados (Mideplan, 2000), por lo que este estudio espera contribuir a un mejor conocimiento anatómico de la zona mandibular.

SUAZO, G. I. C.; MORALES, H. C.A.; CANTÍN, L. M. G.\& ZAVANDO, M. D. A. Biometric aspects of the mandibular canal. Int. J.Morphol., 25(4):811-816, 2007.

SUMMARY: The mandibular canal have as longitudinally course, being related in its average segment to the dental pieces molars and premolars. The knowledge of the relation of the mandibular canal with the dental pieces and cortical bone is of great importance for the practice of the implant therapy. In the present study the relations of the average portion of the canal are analyzed to mandible with cortical mandibular bone pertaining to female sex of between 50 and 65 years obtained of different cemeteries, with coincident anthropometric characterization with the registry. Measurements were made in three levels of the average segment (A-B-C). The results show that the mandibular canal presents a ovoid form and that their diameters diminish as it descends until the mentoniano hole. It was observed, in addition an oblique and descendent passage, coming near to the cortical one to vestibular of the jaw. The importance of the knowledge of the biometrics aspects of the mandibular canal is discussed in female of the selected age group, because they are these patients who have greater necessity of implant therapy.

KEY WORDS: Anatomy; Biometry; Mandibular canal.

\section{REFERENCIAS BIBLIOGRÁFICAS}

Carter, R. B. \& Keen, E. N. Intramandibular course of the inferior alveolar nerve. J. Anat., 108(Pt 3):433-40, 1971.

Farman,A. \& Nortjé C. Panoramic radiographic appearance of the mandibular canal in health and in disease. Panoramic imagine news. 2(2), 2002. www.pancorp.com/newsletter/downloads2/4.pdf

Figún, M. \& Garino, R. Anatomía Odontológica Funcional y Aplicada. 2a . ed. Buenos Aires, El Ateneo, 2001.

Girard, K. Considerations in the management of damage to the mandibular nerve. J.Am.Dent.Assoc., 98:65-71, 1979.

Gowgiel, J. M. The position and course of the mandibular canal. J. Oral Implantol., 18(4):383-5, 1992.

Greenstein, G. \& Tarnow, D. The mental foramen and nerve: Clinical and anatomical factors related to dental implant placement: A literature review. J. Periodontol., 77(12):1933-43, 2006.

Kemkes-Grottenthaler, A.; Löbig, F. \& Stock, F. Mandibular ramus flexure and gonial eversion as morphologic indicators of sex. Homo, 53(2):97-111, 2002.

Kondo, S. \& Townsend, G. C. Sexual dimorphism in crown units of mandibular deciduous and permanent molars in Australian aborigines. Homo, 55(1-2):53-64, 2004.

Latarjet, M. \& Ruiz-Liard, A. Atlas de Anatomía. $2^{\text {a }}$. ed. Buenos Aires, Panamericana, 2004.
Levine, M. H.; Goddard, A. L. \& Dodson, T. B. Inferior alveolar nerve canal position: a clinical and radiographic study. J. Oral. Maxillofac. Surg., 65(3):470-4, 2007.

Lindhe, J. Periodontología Clínica e Implantológica. $4^{\mathrm{a}}$. ed. Buenos Aires, Panamericana, 2005

Ministerio de Planificación y Cooperación (Mideplan). Análisis de la VIII encuesta de caracterización socioeconómica nacional (CASEN). Documento $\mathrm{N}^{\circ} 5$, Situación de Salud en Chile, 2000.

Misch, C. E. Implantología contemporánea. Barcelona, Mosby-Doyma, 1994.

Misch, C. E. Prótesis dental sobre implantes. Barcelona, Elsevier, 2006.

Reiser, G. M.; Manwaring, J. D. \& Damoulis, P. D. Clinical significance of the structural integrity of the superior aspect of the mandibular canal. J. Periodontol., 75(2):322-6, 2004.

Rouvière, H. \& Delmas A. Anatomía Humana Descriptiva, Topográfica y Funcional.10 ${ }^{\mathrm{a}}$. ed. Barcelona, Masson, 1999. V. 3.

Saralaya, V. \& Narayana, K. The relative position of the inferior alveolar nerve in cadaveric hemi-mandibles. Eur. J. Anat., 9(1):49-53, 2005. 
SUAZO, G. I. C.; MORALES, H. C. A.; CANTíN, L. M. G. \& ZAVANDO, M. D. A. Aspectos biométricos del canal mandibular. Int. J. Morphol., 25(4):805-810, 2007.

Soames, R.W. Gray's Anatomy In: Skeletal system. $38^{\text {th }}$ ed. New York, Churchill Livingstone, 1995.

Zachowa, S.; Lamecker, H.; Elsholtz, B. \& StillerM. Is the course of the inferior alveolar nerve deducible from the shape of the mandible? Int. J. CARS., 1:415-19, 2006.
Dirección para correspondencia:

Dr. Iván Suazo Galdames

Unidad de Anatomía Normal

Universidad de Talca

Avenida Lircay s/n Oficina N 104

Talca - CHILE

Fono 56-71-201682

Email: isuazo@utalca.cl

Received: 13-06-2007

Accepted: 28-08-2007 